\title{
Renal Tubular Acidosis Following Topiramate
}

\author{
Huey Kuan Tan ${ }^{\mathrm{a}, \mathrm{b}}$, Supreeth Rudrappa ${ }^{\mathrm{a}}$, Chun Hong Tang ${ }^{\mathrm{a}}$
}

\begin{abstract}
A 20-year-old woman presented with severe lactic acidosis. All investigations have proven negative until we discover the culprit - topiramate, which she takes for cluster headaches.
\end{abstract}

Keywords: Acidosis; Topiramate; Hyperlactataemia

\section{Introduction}

Renal tubular acidosis (RTA) is a medical condition that involves an accumulation of acid in the body due to a failure of the kidneys to appropriately acidify the urine. When blood is filtered through the kidney, the filtrate passes through the tubules of nephrons, allowing for exchange of solutes before it drains into the bladder as urine.

Metabolic acidosis that results from RTA may be caused either by failure to recover sufficient bicarbonate ions from the filtrate in the proximal tubule or by insufficient secretion of hydrogen ions into the distal tubule. There are several different types of RTA, which all have different causes and presentations.

Distal RTA is the classical form of RTA. It is characterized by a failure of acid secretion by the cortical collecting duct of distal nephron. This failure of acid secretion leads to an inability to excrete hydrogen, while potassium cannot be reabsorbed, consequently a tendency towards acidosis.

Topiramate is licensed for the treatment of epilepsy and for migraine prophylaxis, but is also used off-licence for other indications. With the increasing use of topiramate, it is worth noting that reports have emerged that topiramate can cause metabolic acidosis. It does this by impairing both the reabsorption of filtered bicarbonates by the proximal renal tubule and

Manuscript accepted for publication November 13, 2014

${ }^{a}$ Queens Medical Centre, Nottingham University Hospitals, UK

${ }^{\mathrm{b}}$ Corresponding Author: Huey Kuan Tan, Queens Medical Centre, Nottingham

University Hospitals, UK. Email: hueytan@nhs.net

doi: http://dx.doi.org/10.14740/jmc1984w the excretion of hydrogen ions by the distal renal tubule.

This condition is termed mixed RTA. Topiramate-induced RTA can make patients acutely ill, and chronically, can lead to nephrolithiasis and osteoporosis. There is no proven method for predicting or preventing the effect of topiramate on acidbase balance, but for patients with persistent RTA, topiramate should usually be discontinued and replaced with alternatives.

\section{Case Report}

There have been a handful of published cases of topiramate resulting in metabolic acidosis in some patients. Burmeister et al [1] reported a case of a 46-year-old patient who was prescribed topiramate for vertigo and developed metabolic acidosis with a $\mathrm{pH}$ of 7.31 and serum bicarbonates of 8.9. Similarly, Ozer and Altunkaya [2] reported a case of a 58-year-old patient on topiramate for refractory temporal lobe epilepsy who developed metabolic acidosis with a $\mathrm{pH}$ of 7.29 and serum bicarbonates of $20 \mathrm{mM}$.

We report the case of a 20-year-old woman who presented with anaphylactic rash following clarithromycin prescribed by GP for her chest infection. She was fit and well, took topiramate for cluster headaches, sertraline and amitriptyline for depression and had no known allergies. There was no history of alcohol or any other substance abuse.

She continued to feel unwell with palpitations, nausea and headaches despite being treated for anaphylaxis. Rash has completely resolved. The patient was afebrile and had normal white cell count and plasma CRP as well as normal liver parameters. Subsequent blood tests showed metabolic acidosis and hyperlactatemia. The arterial $\mathrm{pH}$ was 7.29 , lactate was 4.8 , and bicarbonates was $12.5 \mathrm{mM}$. Supportive treatment was initiated with fluid resuscitation after which patient's lactate level came down, but just barely.

On this occasion, when her topiramate was titrated down, her acid-base values slowly returned towards normal during the following 14 days of hospital stay.

\section{Discussion}

Drug-induced RTA is considered to be common. However, topiramate being the cause for RTA is rare. The exclusion of 
other possible etiological factors combined with the intake of antibiotics with topiramate make these drugs suspect as causative agents. The reported case of RTA was most likely druginduced by topiramate prescribed for cluster headache. Other etiological factors, i.e. tissue ischemia, diabetes and pancreatitis, were excluded.

In conclusion, due to the coincidence in time and exclusion of other possible etiological factors such as diabetes, pancreatitis, HIV and SLE, the reported case of severe metabolic acidosis with resistant hyperlactatemia was most likely drug-induced [3]. Since introduction of topiramate, although uncommonly, has previously been reported to precipitate metabolic acidosis. At present, there are no published prospective systematic studies of the effect of topiramate on serum $\mathrm{pH}$ and these cases indicate clinical need for studies of this nature.

\section{Competing Interests}

None declared.

\section{References}

1. Burmeister JE, Pereira RR, Hartke EM, Kreuz M. Topiramate and severe metabolic acidosis: case report. Arq Neuropsiquiatr. 2005;63(2B):532-534.

2. Ozer Y, Altunkaya H. Topiramate induced metabolic acidosis. Anaesthesia. 2004;59(8):830.

3. Mirza N, Marson AG, Pirmohamed M. Effect of topiramate on acid-base balance: extent, mechanism and effects. Br J Clin Pharmacol. 2009;68(5):655-661. 